\title{
A Decade of GRB Follow-Up by BOOTES in Spain (2003-2013)
}

\author{
Martin Jelínek, ${ }^{1,2}$ Alberto J. Castro-Tirado,, ${ }^{2,3}$ Ronan Cunniffe, ${ }^{2}$ Javier Gorosabel, $, 4,5$ \\ Stanislav Vítek, ${ }^{6}$ Petr Kubánek, ${ }^{7,8}$ Antonio de Ugarte Postigo, ${ }^{2}$ Sergey Guziy, ${ }^{2}$ \\ Juan C. Tello, ${ }^{2}$ Petr Páta, ${ }^{6}$ Rubén Sánchez-Ramírez, ${ }^{2}$ Samantha Oates, ${ }^{2}$ Soomin Jeong, ${ }^{2,9}$ \\ Jan Štrobl, ${ }^{1}$ Sebastián Castillo-Carrión, ${ }^{10}$ Tomás Mateo Sanguino, ${ }^{11}$ Ovidio Rabaza, ${ }^{12}$ \\ Dolores Pérez-Ramírez, ${ }^{13}$ Rafael Fernández-Muñoz, ${ }^{14}$ Benito A. de la Morena Carretero, ${ }^{15}$ \\ René Hudec, ${ }^{1,6}$ Víctor Reglero, ${ }^{8}$ and Lola Sabau-Graziati ${ }^{16}$ \\ ${ }^{1}$ Astronomický Ústav AV ČR, Ondřejov (ASÚ AV ČR), Ondřejov, Czech Republic \\ ${ }^{2}$ Instituto de Astrofísica de Andalucía- (IAA-) CSIC, 18008 Granada, Spain \\ ${ }^{3}$ Departamento de Ingeniería de Sistemas y Automática (Unidad Asociada al CSIC), Universidad de Málaga, 29010 Málaga, Spain \\ ${ }^{4}$ Unidad Asociada Grupo Ciencia Planetarias UPV/EHU-IAA/CSIC, Departamento de Física Aplicada I, \\ E.T.S. de Ingeniería, Universidad del País Vasco (UPV)/EHU, Alameda de Urquijo s/n, 48013 Bilbao, Spain \\ ${ }^{5}$ Ikerbasque, Basque Foundation for Science, Alameda de Urquijo 36-5, 48008 Bilbao, Spain \\ ${ }^{6}$ České Vysoké Učení Technické, Fakulta Elektrotechnická (ČVUT-FEL), Praha, Czech Republic \\ ${ }^{7}$ Fyzikální ústav AV ČR, Na Slovance 2, 18221 Praha 8, Czech Republic \\ ${ }^{8}$ Image Processing Laboratory, Universidad de Valencia, Burjassot, Valencia, Spain \\ ${ }^{9}$ Institute for Science and Technology in Space, Natural Science Campus, Sungkyunkwan University, Suwon 440-746, Republic of Korea \\ ${ }^{10}$ Universidad de Málaga, Campus de Teatinos, Málaga, Spain \\ ${ }^{11}$ Departamento de Ingeniería de Sistemas y Automática, Universidad de Huelva, E.P.S. de La Rábida, Huelva, Spain \\ ${ }^{12}$ Department of Civil Engineering, University of Granada, 18071 Granada, Spain \\ ${ }^{13}$ Universidad de Jaén, Campus las Lagunillas, 23071 Jaén, Spain \\ ${ }^{14}$ Instituto de Hortofruticultura Subtropical y Mediterránea "La Mayora" (IHSM-CSIC), Algarrobo, 29750 Málaga, Spain \\ ${ }^{15}$ Estación de Sondeos Atmosféricos (ESAt) de El Arenosillo (CEDEA-INTA), Mazagón, Huelva, Spain \\ ${ }^{16}$ División de Ciencias del Espacio, INTA, Torrejón de Ardoz, Madrid, Spain
}

Correspondence should be addressed to Martin Jelínek; mates@iaa.es

Received 11 March 2016; Accepted 17 August 2016

Academic Editor: Dean Hines

Copyright ( 2016 Martin Jelínek et al. This is an open access article distributed under the Creative Commons Attribution License, which permits unrestricted use, distribution, and reproduction in any medium, provided the original work is properly cited.

This article covers ten years of GRB follow-ups by the Spanish BOOTES stations: 71 follow-ups providing 23 detections. Follow-ups by BOOTES-1B from 2005 to 2008 were given in a previous article and are here reviewed and updated, and additional detection data points are included as the former article merely stated their existence. The all-sky cameras CASSANDRA have not yet detected any GRB optical afterglows, but limits are reported where available.

Dedicated to the memory of Dolores Pérez-Ramírez and Javier Gorosabel, who passed away while this paper was in preparation

\section{Introduction}

Ever since the discovery of Gamma-ray bursts (GRB) in 1967 [1], it was hoped to discover their counterparts at other wavelengths. The early GRB-related transient searching methods varied (wide-field optical systems as well as deep searches were being employed) but, given the coarse gammaray-based GRB localizations provided, generally lacked either 
sensitivity or good reaction time. The eventual discovery of GRB optical counterparts was done only when an X-ray follow-up telescope was available on the BeppoSAX satellite [2]. The optical afterglow could then be searched for with a large telescope in a small errorbox provided by the discovery of the X-ray afterglow. The first optical afterglow of a gammaray burst was discovered this way in 1997 [3].

Since then, astronomers have been trying to minimize the time delay between receiving the position and the start of observations-by both personal dedication and by automating the telescope reaction. The ultimate step in automation, to minimize the time delay, is a full robotization of the observatory to eliminate any human intervention in the followup process. This way, the reaction time can be minimized from $\sim 10$-minute limit that can be achieved with a human operated telescope to below 10 seconds. With improvements in computational methods and in image processing speed, blind (non-follow-up) wide-field methods are starting to be practical in the search for optical transients. Although limited in magnitude range, they have already provided important observations of the optical emission simultaneous to the gamma-ray production of a GRB [4].

Since 1997, the robotic telescope network BOOTES has been part of the effort to follow up gamma-ray burst events [5]. As of now, the network of robotic telescopes BOOTES consists of six telescopes around the globe, dedicated primarily to GRB afterglow follow-up. We present the results of our GRB follow-up programme by two telescopes of the network-BOOTES-1B and BOOTES-2-and by the respective stationary very-wide-field cameras (CASSANDRA). This text covers eleven years of GRB follow-ups: 71 follow-ups providing 21 detections.

Different instruments have been part of BOOTES during the years in question: a $30 \mathrm{~cm}$ telescope which was used for most of the time at BOOTES-1 station but at periods also at BOOTES-2, the fast-moving $60 \mathrm{~cm}$ telescope at BOOTES2 (Telma), and also two all-sky cameras, CASSANDRA1 at BOOTES-1 and CASSANDRA2 at BOOTES-2. Results from CASSANDRAs are included where available, without paying attention to the complete sample.

This article is a follow-up of a previous article, that is, Jelínek et al. [6], which provided detailed description of evolution of BOOTES-1B, and analysis of efficiency of a system dedicated to GRB follow-up based on real data obtained during four years between 2005 and 2008. This work is a catalogue of BOOTES-1B and BOOTES-2 GRB observations between 2003 and 2013; it is complete in providing information about successfully followed up events but does not provide analysis of missed triggers as did the previous article.

1.1. BOOTES-1B. BOOTES-1 observatory is located at the atmospheric sounding station at El Arenosillo, Huelva, Spain (at lat.: $37^{\circ} 06^{\prime} 14^{\prime \prime} \mathrm{N}$, long.: $06^{\circ} 44^{\prime} 02^{\prime \prime} \mathrm{W}$ ). Over time, distinct system configurations were used, including also two 8-inch $\mathrm{S}-\mathrm{C}$ telescopes, as described in Jelínek et al. [6]; the primary instrument of BOOTES-1B is a $D=30 \mathrm{~cm}$ SchmidtCassegrain optical tube assembly with a CCD camera. Prior to June 15, 2007, Bessel VRI filters were being used as noted with the observations, any observations obtained after this date have been obtained without filter ( $C$ or clear). We calibrate these observations against $R$-band, which, in the case of no color evolution of the optical counterpart, is expected to result in a small $(\sim 0.1 \mathrm{mag})$ constant offset in magnitude.

1.2. BOOTES-2. BOOTES-2 is located at CSIC's experimental station La Mayora (Instituto de Hortofruticultura Subtropical y Mediterránea- (IHSM-) CSIC) (at lat.: $36^{\circ} 45^{\prime} 33^{\prime \prime} \mathrm{N}$, long.: $\left.04^{\circ} 02^{\prime} 27^{\prime \prime} \mathrm{W}\right), 240 \mathrm{~km}$ from BOOTES-1. It was originally equipped with an identical $30 \mathrm{~cm}$ Schmidt-Cassegrain telescope to that at BOOTES-1B. In 2007 the telescope was upgraded to a lightweight $60 \mathrm{~cm}$ Ritchey-Chrètien telescope on a fast-slewing NTM-500 mount, both provided by Astelco. The camera was upgraded at the same time to an Andor iXon $1024 \times 1024$ EMCCD, and in 2012 the capabilities were extended yet again to low resolution spectroscopy, by the installation of the imaging spectrograph COLORES of our own design and construction [7]. Bessel magnitudes are calibrated to Vega system, SDSS to AB.

\section{Optical Follow-Up of GRB Events}

Here we will detail the individual results for each of the 23 events followed up and detected in 2003-2013. Each GRB is given a short introductory paragraph as a reminder of the basic observational properties of the event. Although we do not discuss the properties at other wavelengths, we try to include a comprehensive reference of literature relevant to each burst. As GCN reports usually summarise the relevant GCN circular traffic, we have omitted the raw GCN circulars except for events for which a GCN report or other more exhaustive paper is unavailable.

Further 48 follow-ups which resulted in detection limits are included in Tables 1 and 2 but are not given any further attention.

One by one, we show all the successful follow-ups that these telescopes have performed during the first ten years of the Swift era and since the transition of the BOOTES network to the RTS-2 [14] observatory control system, which was for the first time installed at BOOTES-2 in 2003 and during the summer of 2004 at BOOTES-1.

GRB 050525A (A Bright Low-Redshift $(z=0.606)$ Localized by Swift [15]). Plenty of optical observations were obtained, including the signature of the associated supernova sn $2005 \mathrm{nc}$ $[8,16]$.

GRB 050525A was the first BOOTES-1B burst for which a detection was obtained. The telescope started the first exposure $28 \mathrm{~s}$ after receiving the notice, $383 \mathrm{~s}$ after the GRB trigger. An optical afterglow with $V \simeq 16$ was detected. A weak detection of a bright GRB implied a reexamination of observing strategies employed by BOOTES. The largest, $30 \mathrm{~cm}$ telescope was changed to make $R$-band imaging instead of using the field spectrograph to greatly improve sensitivity in terms of limiting magnitude. The $20 \mathrm{~cm}$ telescopes were still observing with $V+I$ filters (for details see [6]); see Table 3 . 
TABLE 1: BOOTES-1B GRBs in a table.

\begin{tabular}{|c|c|c|c|c|}
\hline GRB & $\Delta T$ & Number of points & Result & Ref. \\
\hline 030913 & $2 \mathrm{~h}$ & & $V>17.5, C>12$ & \\
\hline 050215B & $22 \mathrm{~m}$ & & $V>16.5, I>15.0$ & \\
\hline 050505 & $47 \mathrm{~m}$ & & $V>19$ & \\
\hline 050509A & $64 \mathrm{~m}$ & & $V>14.9$ & \\
\hline 050509B & $62 \mathrm{~s}$ & & $V>11.5$ & \\
\hline 050525A & $12 \mathrm{~m}^{\dagger}$ & 1 & $16.5 \pm 0.4$ & {$[8]$} \\
\hline 050528 & $71 \mathrm{~s}$ & & $V>13.8, I>13.0$ & \\
\hline 050824 & $10 \mathrm{~m}$ & 4 & $R=18.2 \pm 0.3$ & [9] \\
\hline 050904 & $2 \mathrm{~m}$ & & $R>18.2$ & {$[10]$} \\
\hline 050922C & $4 \mathrm{~m}$ & 3 & $R=14.6 \pm 0.4$ & \\
\hline 051109A & $55 \mathrm{~s}$ & 6 & $R=15.7 \pm 0.4$ & \\
\hline 051211B & $42 \mathrm{~s}$ & & $R>14$ & \\
\hline 051221B & $4 \mathrm{~m}$ & & $R>16$ & \\
\hline 060421 & $61 \mathrm{~s}$ & & $R>14$ & \\
\hline 061110B & $11 \mathrm{~m}$ & & $R>18$ & \\
\hline 071101 & $55 \mathrm{~s}$ & & $C>17.0$ & \\
\hline 071109 & $59 \mathrm{~s}$ & & $C>13.0$ & \\
\hline 080330 & $6 \mathrm{~m}$ & 6 & $C=16.5 \pm 0.2$ & \\
\hline 080413A & $61 \mathrm{~s}$ & 61 & $C \simeq 13.3$ & \\
\hline 080430 & $34 \mathrm{~s}$ & 1 & $C \simeq 15.5$ & \\
\hline 080603B & $1 \mathrm{~h}$ & 11 & $C \simeq 17.4$ & {$[11]$} \\
\hline 080605 & $44 \mathrm{~s}$ & 28 & $C \simeq 14.7$ & {$[12]$} \\
\hline 081003B & $41 \mathrm{~s}$ & & $C>17.6$ & \\
\hline 090313 & $12 \mathrm{~h}$ & 1 & $C \simeq 18.3$ & \\
\hline 090519 & $99 \mathrm{~s}$ & & $C>17.6$ & \\
\hline 090813 & $53 \mathrm{~s}$ & 1 & $C \simeq 17.9$ & \\
\hline 090814A & $3 \mathrm{~m}^{\dagger}$ & & $C>15.8$ & \\
\hline 090814B & $53 s^{\dagger}$ & & $C>17.5$ & \\
\hline 090817 & $24 \mathrm{~m}$ & & $C>16.7$ & \\
\hline $100906 \mathrm{~A}$ & $106 s$ & & $C>16.5$ & \\
\hline $110205 \mathrm{~A}$ & $102 \mathrm{~s}$ & 16 & $C \sim 14$ & {$[13]$} \\
\hline $110212 \mathrm{~A}$ & $50 \mathrm{~s}$ & & $C>13.0$ & \\
\hline $110213 \mathrm{~A}$ & $15 \mathrm{~h}$ & 1 & $C=18.3 \pm 0.2$ & \\
\hline $110411 \mathrm{~A}$ & $24 \mathrm{~s}$ & & $C>17.8$ & \\
\hline 111016 A & $1.25 \mathrm{~h}$ & & $C>17.8$ & \\
\hline $120326 \mathrm{~A}$ & $40 \mathrm{~m}$ & 1 & $C \sim 19.5$ & \\
\hline $120327 \mathrm{~A}$ & $41 \mathrm{~m}^{\dagger}$ & 6 & $C=17.5$ & \\
\hline $120328 \mathrm{~A}$ & $7.5 \mathrm{~m}$ & & $C>16$ & \\
\hline $120521 \mathrm{C}$ & $11.7 \mathrm{~m}$ & & $C>20.5$ & \\
\hline 120711B & $107 \mathrm{~s}$ & & $C>18.2$ & \\
\hline $120729 \mathrm{~A}$ & $10 \mathrm{~h}$ & & $C>19.0$ & \\
\hline $121017 \mathrm{~A}$ & $79 \mathrm{~s}$ & & $C>19.0$ & \\
\hline $121024 \mathrm{~A}$ & $40 \mathrm{~m}$ & 1 & $C=18.2 \pm 0.5$ & \\
\hline $121209 A$ & $42 s^{\dagger}$ & & $C>16.5$ & \\
\hline 130122A & $28 \mathrm{~m}$ & & $C>18.4$ & \\
\hline
\end{tabular}

Note. $\dagger$ marks alerts covered in real time by wide-field camera CASSANDRA1.

This burst was covered in real time by both all-sky cameras of BOOTES (CASSANDRA1 and CASSANDRA2), providing an unfiltered limit of $>9.0$ [17].
TABLE 2: BOOTES-2 GRBs in a table.

\begin{tabular}{|c|c|c|c|c|}
\hline GRB & $\Delta T$ & Number of points & Result & Ref. \\
\hline 080603B & & 20 & $R \simeq 17.4$ & {$[11]$} \\
\hline 080605 & & 5 & $R \simeq 14.7$ & {$[12]$} \\
\hline 090817 & $145 \mathrm{~s}$ & & $R>18.3$ & \\
\hline 090904A & $86 s$ & & $R>16.1$ & \\
\hline 091202 & $5.5 \mathrm{~h}$ & & $R>18.3$ & \\
\hline 100219A & $6.3 \mathrm{~h}$ & & $C>18.3$ & \\
\hline $100418 \mathrm{~A}$ & $1.8 \mathrm{~h}$ & 11 & $C=19.3$ & \\
\hline $100522 \mathrm{~A}$ & $625 \mathrm{~s}$ & & $C>15.5$ & \\
\hline $100526 \mathrm{~A}$ & $4 \mathrm{~h}$ & & $r^{\prime}>14$ & \\
\hline $100614 \mathrm{~A}$ & $6.9 \mathrm{~m}$ & & $C>18$ & \\
\hline $100901 \mathrm{~A}$ & $10 \mathrm{~h}$ & 10 & $C=17.52 \pm 0.08$ & \\
\hline $100915 \mathrm{~A}$ & $106 \mathrm{~s}$ & & $C>16.5$ & \\
\hline $101020 \mathrm{~A}$ & $5.1 \mathrm{~h}$ & & $r^{\prime}>18.0$ & \\
\hline 101112A & $595 \mathrm{~s}$ & 15 & $C=15.5$ & \\
\hline $110106 \mathrm{~B}$ & $10.3 \mathrm{~m}$ & & $C>16.5$ & \\
\hline $110205 \mathrm{~A}$ & $15 \mathrm{~m}$ & 13 & $R \sim 14$ & {$[13]$} \\
\hline $110212 \mathrm{~A}$ & $32 \mathrm{~m}$ & & $R>16.5$ & \\
\hline $110223 \mathrm{~A}$ & $228 s$ & & $R>17.6$ & \\
\hline $120729 \mathrm{~A}$ & $13.25 \mathrm{~h}$ & & $R>19.4$ & \\
\hline $120805 \mathrm{~A}$ & $25 \mathrm{~m}$ & & $R>18.5$ & \\
\hline $120816 \mathrm{~A}$ & $66 \mathrm{~m}$ & & $R>18$ & \\
\hline $121001 \mathrm{~A}$ & $32 \mathrm{~m}$ & & $I>19.7$ & \\
\hline $121017 \mathrm{~A}$ & $3 \mathrm{~m}$ & & $C>18.5, i^{\prime}>19.5$ & \\
\hline 130418A & $1.5 \mathrm{~h}$ & 21 & $C=16.8 \pm 0.06$ & \\
\hline $130505 \mathrm{~A}$ & $11.94 \mathrm{~h}$ & 1 & $R_{C}=19.26 \pm 0.06$ & \\
\hline $130606 \mathrm{~A}$ & $13 \mathrm{~m}$ & 21 & $i^{\prime}=16.7 \pm 0.3$ & \\
\hline $130608 \mathrm{~A}$ & $2.3 \mathrm{~h}$ & & $C>18.8$ & \\
\hline $130612 \mathrm{~A}$ & $4.8 \mathrm{~m}$ & & $C>18.6$ & \\
\hline $130806 \mathrm{~A}$ & $40 \mathrm{~s}$ & & $C>18.3$ & \\
\hline $131202 \mathrm{~A}$ & $4.25 \mathrm{~h}$ & & $i^{\prime}>19.7$ & \\
\hline
\end{tabular}

TABLE 3: GRB 050525A: observing log of BOOTES-1B.

\begin{tabular}{lcccc}
\hline$\Delta T[\mathrm{~h}]$ & $\exp [\mathrm{s}]$ & $\mathrm{mag}$ & $\mathrm{dmag}$ & Filter \\
\hline 0.195 & $39 \times 10 \mathrm{~s}$ & 16.51 & 0.39 & $R$ \\
\hline
\end{tabular}

Note. Published by Resmi et al. [8].

BOOTES observation of this GRB is included in Resmi et al. [8].

GRB 050824 (A Dim Burst Detected by Swift). The optical afterglow of this GRB is discovered with the $1.5 \mathrm{~m}$ telescope at OSN; redshift $z=0.83$ as determined by VLT [9].

BOOTES-1B was the first telescope to observe this optical transient, starting $636 \mathrm{~s}$ after the trigger with $R \simeq 17.5$. The weather was not stable and the focus not perfect, but BOOTES-1B worked as expected. In the end, several hours of data were obtained. BOOTES observation of this GRB is included in Sollerman et al. [9]; see Table 4 and Figure 1.

GRB 050922C. A Swift short and intense long burst [18, 19] was observed also by HETE2 [20]. Optical afterglow is mag $15 ; z=2.198$ [21]. 


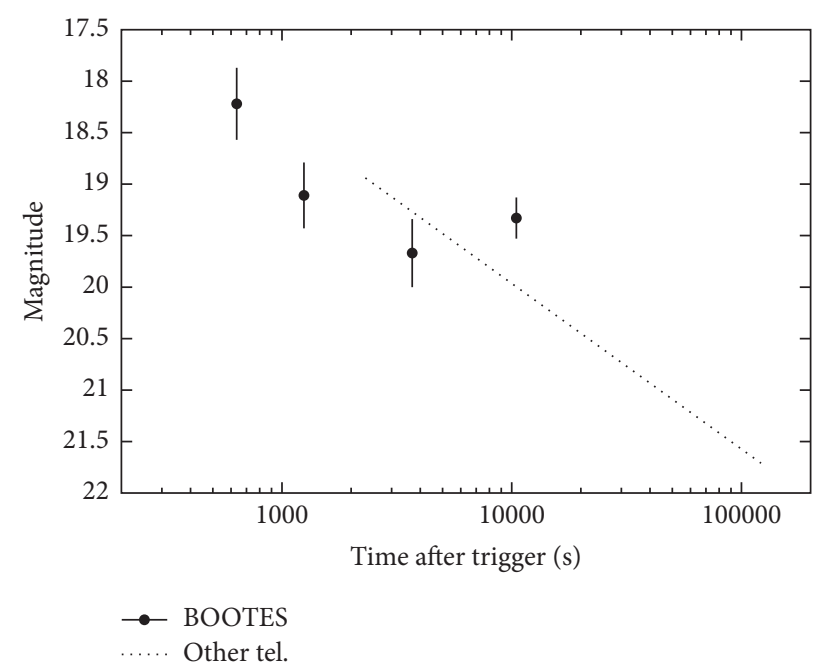

FIgURE 1: The optical light curve of GRB 050824; the optical light curve represents the behaviour seen by Sollerman et al. [9].

TABLE 4: GRB 050824: observing log of BOOTES-1B.

\begin{tabular}{lcccc}
\hline$\Delta T[\mathrm{~h}]$ & $\exp [\mathrm{s}]$ & mag & dmag & Filter \\
\hline 0.1763 & $2 \times 300 \mathrm{~s}$ & 18.22 & 0.35 & $R$ \\
0.3462 & $8 \times 300 \mathrm{~s}$ & 19.11 & 0.32 & $R$ \\
1.0249 & $22 \times 300 \mathrm{~s}$ & 19.67 & 0.33 & $R$ \\
2.9091 & $31 \times 300 \mathrm{~s}$ & 19.33 & 0.20 & $R$ \\
\hline
\end{tabular}

Note. Published by Sollerman et al. [9].

TABLE 5: GRB 050922C: observing log of BOOTES-1B.

\begin{tabular}{lcccc}
\hline$\Delta T[\mathrm{~h}]$ & $\exp [\mathrm{s}]$ & mag & dmag & Filter \\
\hline 0.0694 & 40 & 14.58 & 0.35 & $R$ \\
0.3752 & 900 & 17.01 & 0.39 & $R$ \\
0.6193 & 900 & 18.53 & 0.59 & $R$ \\
\hline
\end{tabular}

Due to clouds, the limiting magnitude of BOOTES-1B dropped from $\sim 17.0$ for a $30 \mathrm{~s}$ exposure to merely 12.9. The afterglow was eventually detected with the $R$-band camera (at the $30 \mathrm{~cm}$ telescope) during gaps between passing clouds. The first weak detection was obtained $228 \mathrm{~s}$ after the GRB trigger and gave $R \simeq 14.6$; see Table 5 .

GRB 051109A (A Burst Detected by Swift [23]). The optical afterglow was mag $\sim 15$, and the redshift was determined to be $z=2.346$ [24]. The optical lightcurve was published by Mirabal et al. [22].

At BOOTES-1B the image acquisition started $54.8 \mathrm{~s}$ after the burst with the $30 \mathrm{~cm}$ telescope in $R$-band and one of the $20 \mathrm{~cm}$ telescopes in $I$-band [25]. There were still a number of performance problems-most importantly synchronization between cameras such that when the telescope position was to be changed, both cameras had to be idle. As the $30 \mathrm{~cm}$ telescope was taking shorter exposures, extra exposures could have been made while waiting for the longer exposures being taken at the $20 \mathrm{~cm}$ to finish. The $20 \mathrm{~cm}$ detection is, after critical revision, only at the level of $2-\sigma$. The $R$-band
TABLE 6: GRB 051109A: observing log of BOOTES-1B.

\begin{tabular}{lcccc}
\hline$\Delta T[\mathrm{~s}]$ & $\exp [\mathrm{s}]$ & mag & dmag & Filter \\
\hline 59.7 & 10 & 15.67 & 0.35 & $R$ \\
122.2 & 74 & 16.02 & 0.19 & $R$ \\
257.9 & 41 & 16.65 & 0.41 & $R$ \\
756.6 & 205 & 17.18 & 0.22 & $R$ \\
1021.5 & 313 & 17.68 & 0.26 & $R$ \\
508.4 & 908 & 16.98 & 0.54 & $I$ \\
\hline
\end{tabular}

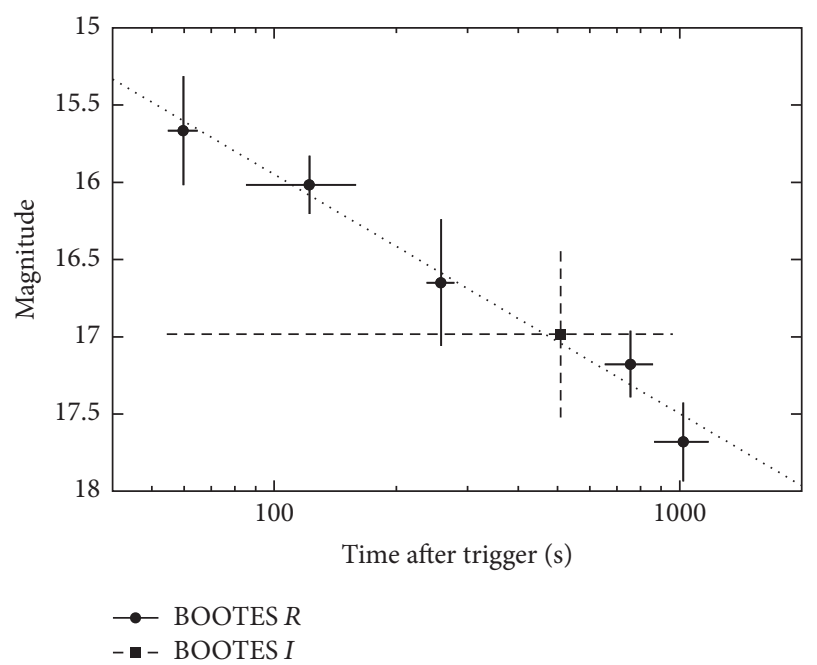

FIgURE 2: The optical light curve of GRB 051109A. The dotted line represents the optical decay observed by Mirabal et al. [22].

observation shows the object until about 20 minutes after the GRB, when it becomes too dim to measure in the vicinity of a $17.5 \mathrm{~m}$ nearby star. Mean decay rate observed by BOOTES is $\alpha=0.63 \pm 0.06\left(F_{\mathrm{opt}} \sim t^{-\alpha}\right)$.

The relatively shallow decay observed by BOOTES is in close agreement with what was observed several minutes later by the $2.4 \mathrm{~m} \mathrm{MDM}(\alpha=0.62 \pm 0.03)$ and according to an unofficial report [26] there was a decay change later, by about $3 \mathrm{~h}$ after the burst to $\alpha=0.89 \pm 0.05$; see Table 6 and Figure 2 .

GRB 080330 (A Rather Bright Long Burst Detected by Swift). Afterglow was reported to be detected by UVOT, TAROT, ROTSE-III, Liverpool Telescope, and GROND. Spectroscopic redshift was measured as $z=1.51$ by the NOT [28].

This GRB happened during the first day recommissioning of BOOTES-1B after its move from the BOOTES-2 site at La Mayora. The GCN client was not yet operational and at the time of the GRB we were focusing the telescope. The first image was obtained $379 \mathrm{~s}$ after the GRB trigger and the optical afterglow was detected with magnitude $\sim 16.3$ on the first image. A bug in the centering algorithm caused a loss of part subsequent data. Further detections were obtained starting $21 \mathrm{~min}$ after the GRB when the problem was fixed.

The light curve (as seen by [27]) seems to show an optical flare and then a possible hydrodynamic peak. The data of BOOTES, however, trace only the final part of this 


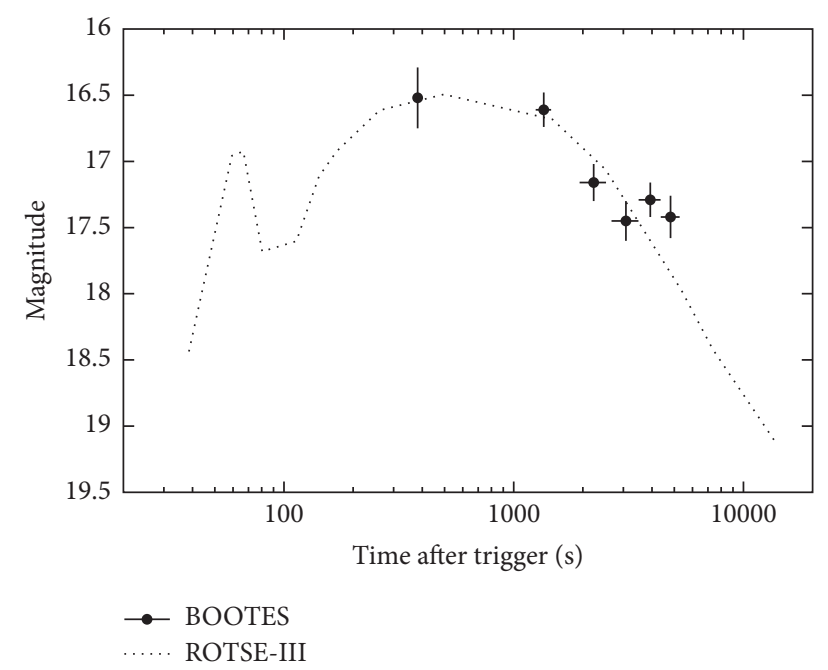

FIgure 3: The optical light curve of GRB 080330. The dotted line shows the light curve as seen by ROTSE-III [27].

TABLE 7: GRB 080330: observing log of BOOTES-1B.

\begin{tabular}{lcccc}
\hline$\Delta T[\mathrm{~h}]$ & $\exp [\mathrm{s}]$ & mag & dmag & Filter \\
\hline 0.1061 & 7 & 16.52 & 0.23 & Clear \\
0.3752 & 210 & 16.61 & 0.13 & Clear \\
0.6193 & 588 & 17.16 & 0.14 & Clear \\
0.8547 & 825 & 17.45 & 0.15 & Clear \\
1.0915 & 862 & 17.29 & 0.13 & Clear \\
1.3384 & 905 & 17.42 & 0.16 & Clear \\
\hline
\end{tabular}

behaviour, where the decay accelerates after passing through the hydrodynamic peak; see Table 7 and Figure 3.

GRB 080413A. A rather bright GRB was detected by Swift and also by Suzaku-WAM; optical afterglow was detected by ROTSE-III [27]; and redshift $z=2.433$ was detected by VLT+UVES [29].

BOOTES-1B started obtaining images of the GRB 080413A just $60.7 \mathrm{~s}$ after the trigger ( $46.3 \mathrm{~s}$ after reception of the alert). An $R \simeq 13.3$ magnitude decaying optical afterglow was found ([30], Jelinek et al., in prep.); see Figure 4.

GRB 080430 (A Burst Detected by Swift). It was a widely observed, low-redshift $z \simeq 0.75$ optical afterglow with a slowly decaying optical afterglow [31]. It was observed also at very high energies by MAGIC without detection [32].

BOOTES-1B obtained the first image of this GRB $34.4 \mathrm{~s}$ after the trigger. An optical transient was detected on combined unfiltered images with a magnitude $\simeq 15.5$ [33].

GRB 080603B. A long GRB localized by Swift is detected also by Konus-Wind and by INTEGRAL [34]. Bright optical afterglow was observed. Extensive follow-up was carried out. Redshift is $z=2.69$ [35].

This GRB happened in Spain during sunset. We obtained first useful images starting one hour after the trigger. An

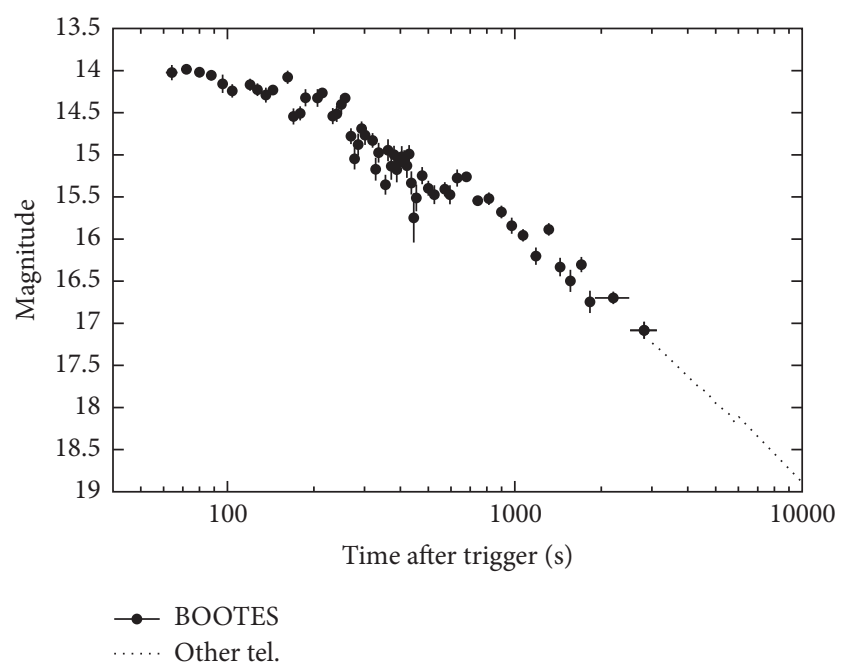

FIGURE 4: The optical light curve of GRB 080413A.

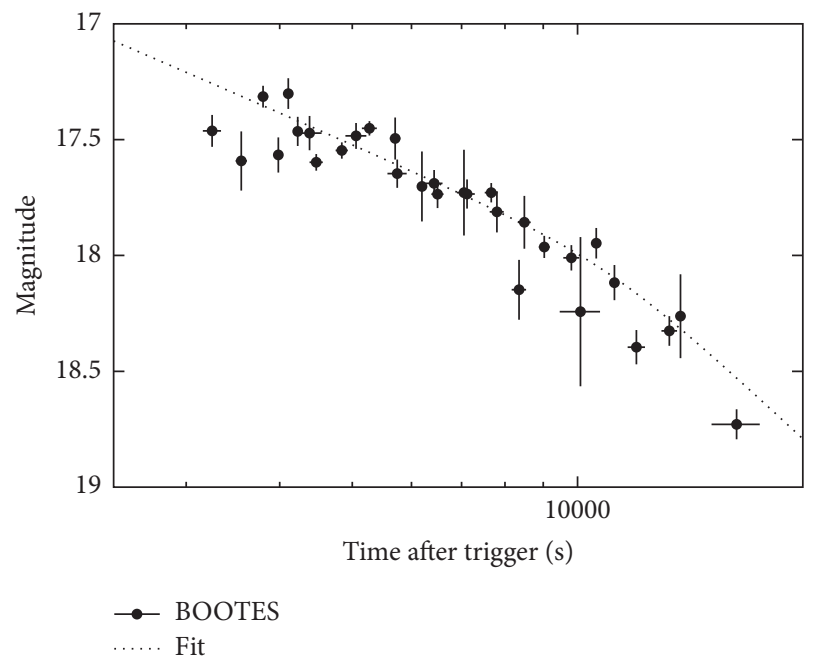

Figure 5: The optical light curve of GRB 080603B [11].

$R \simeq 17.4$ optical transient was detected with both BOOTES$1 \mathrm{~B}$ and BOOTES-2; see Figure 5. BOOTES observation of this GRB is included in Jelínek et al. [11]; see Figure 5.

GRB 080605 (A Long Burst Detected by Swift [36]). The host was found to be a metal enriched star forming galaxy at redshift $z=1.64$ [37] and exhibited the $2175 \AA$ extinction feature [38].

GRB 080605 was observed by both BOOTES-1B (28 photometric points) and BOOTES-2 (5 photometric points) starting $44 \mathrm{~s}$ after the trigger. A rapidly decaying optical afterglow $(\alpha=1.27 \pm 0.04)$ with $R=14.7$ on the first images was found; see Figure 6 . All BOOTES data are included in Jelínek et al. [12]; see Figure 5.

GRB 090313 (GRB by Swift, No Prompt X-Rays [40]). An optical afterglow peaked at $R \sim 15.6$. Extensive optical + infrared follow-up was carried out. The first GRB to be observed 


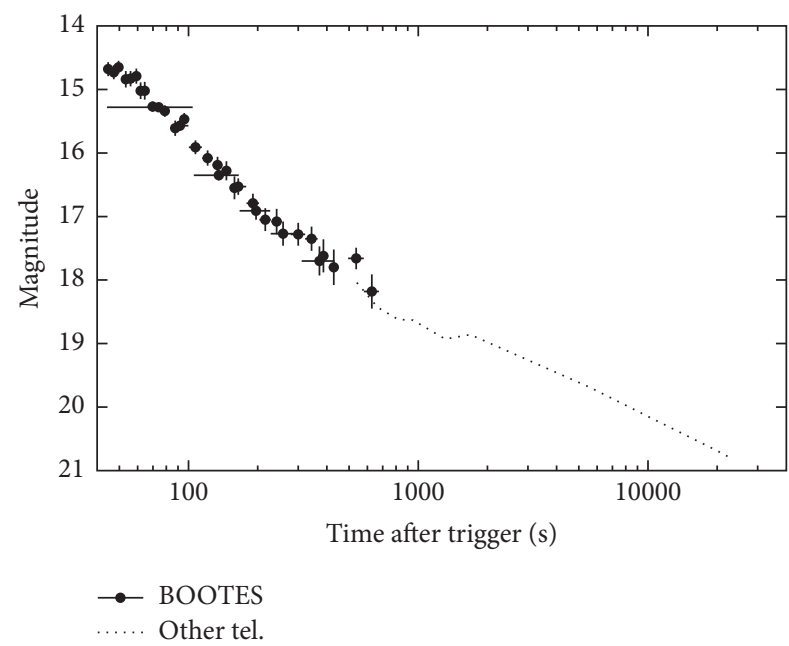

FIgURE 6: The optical light curve of GRB 080605 [12]; the dotted line is behaviour observed by Rumyantsev and Pozanenko [39] and Zafar et al. [38].

TABLE 8: GRB 090813: observing log of BOOTES-1B.

\begin{tabular}{lcccc}
\hline$\Delta T[\mathrm{~h}]$ & $\exp [\mathrm{s}]$ & $\mathrm{mag}$ & dmag & Filter \\
\hline 0.175 & $10 \times 10$ & 17.9 & 0.3 & Clear \\
\hline
\end{tabular}

was detected by X-Shooter. Also it was detected by various observatories in radio. Redshift is $z=3.375$ [41, 42].

The GRB happened during daytime for BOOTES-1B and it was followed up manually. Due to the proximity of the moon and limitations of then-new CCD camera driver, many $2 \mathrm{~s}$ exposures were taken to be combined later. The optical afterglow was detected with magnitude $\sim 18.3 \pm 0.4$ on a $635 \times$ $2 \mathrm{~s}$ (=21 min) exposure with the midtime $11.96 \mathrm{~h}$ after the GRB trigger.

GRB 090813. A long GRB by Swift, suspected of being higher$z$, observed also by Konus-Wind and Fermi-GBM [43]. Optical counterpart was observed by the $1.23 \mathrm{~m}$ telescope at Calar Alto with a magnitude of $I=17.0$ [44].

BOOTES-1B started observation $53 \mathrm{~s}$ after the GRB, taking $10 \mathrm{~s}$ unfiltered exposures. The optical transient was weakly detected on a combined image of $10 \times 10 \mathrm{~s}$ whose exposure mean time was $630 \mathrm{~s}$ after the burst. The optical counterpart was found having $R=17.9 \pm 0.3$. Given that the previous and subsequent images did not show any OT detection, we might speculate about the optical emission peaking at about this time. Also the brightness is much weaker than what might be expected from the detection by Gorosabel et al. [44], supporting the high-redshift origin; see Table 8.

GRB 100418A. A weak long burst was detected by Swift [45] with a peculiar, late-peaking optical afterglow with $z=$ 0.6239 [46]. Also it was detected in radio [47].

The first image of the GRB location was taken by BOOTES-2 at 21:50 UT (40 min after the GRB trigger). The rising optical afterglow was detected for the first time on an image obtained as a sum of 23 images, with an exposure
TABLE 9: GRB 100418A: observing log of BOOTES-2.

\begin{tabular}{lcccc}
\hline$\Delta T[\mathrm{~h}]$ & exp $[\mathrm{s}]$ & mag & dmag & Filter \\
\hline 1.78 & 1638 & 19.785 & 0.215 & Clear \\
2.09 & 597 & 19.127 & 0.127 & Clear \\
2.55 & 534 & 18.774 & 0.087 & Clear \\
2.72 & 656 & 18.668 & 0.073 & Clear \\
3.10 & 239 & 18.706 & 0.106 & Clear \\
3.43 & 238 & 18.759 & 0.189 & Clear \\
4.70 & 3908 & 19.067 & 0.108 & Clear \\
6.19 & 4328 & 18.897 & 0.115 & Clear \\
7.39 & 551 & 18.493 & 0.078 & Clear \\
77.3 & 14830 & 20.475 & 0.202 & Clear \\
125.6 & 12482 & 20.970 & 0.208 & Clear \\
\hline
\end{tabular}

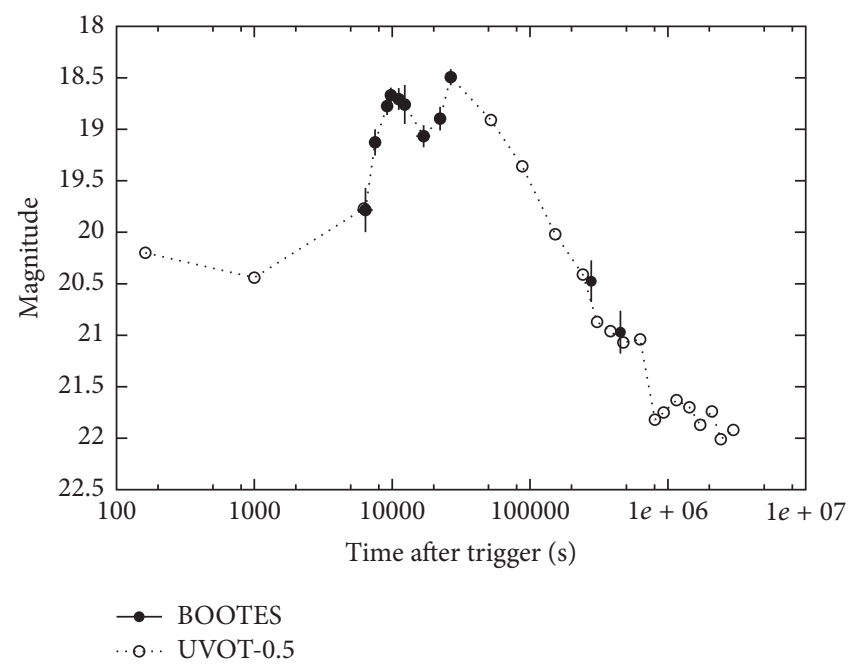

FIgure 7: The bizarre optical light curve of GRB 100418A. Combination of BOOTES and UVOT data [45]. UVOT points were shifted by an arbitrary constant.

midtime 107 minutes after the GRB trigger. The optical emission peaked at magnitude $R=18.7$ another hour later, at an image with the midtime $163 \mathrm{~min}$ after the trigger. A slow decay followed, which permitted us to detect the optical counterpart until 8 days after the GRB.

Because of a mount problem, many images were lost (pointed somewhere else) and the potential of the telescope was not fully used. Eventually, after combining images when appropriate, 11 photometric points were obtained. A rising part of the optical afterglow was seen that way; see Table 9 and Figure 7.

GRB 100901A (A Long Burst from Swift). Bright, slowly decaying optical afterglow was discovered by UVOT. Redshift is $z=1.408$. It was detected also by SMA at $345 \mathrm{GHz}$ [48-50].

The burst happened in daytime in Spain and the position became available only almost ten hours later after the sunset. The afterglow was still well detected with magnitude $R \simeq 17.5$ at the beginning. BOOTES-2 had some problems with CCD cooling, and some images were useless. The afterglow was 
TABLE 10: GRB 100901A: observing log of BOOTES-2.

\begin{tabular}{lcccc}
\hline$\Delta T[\mathrm{~h}]$ & exp $[\mathrm{s}]$ & mag & dmag & Filter \\
\hline 10.202 & 268 & 17.52 & 0.08 & $R$ \\
10.719 & 415 & 17.61 & 0.07 & $R$ \\
11.230 & 354 & 17.67 & 0.09 & $R$ \\
11.734 & 238 & 17.99 & 0.16 & $R$ \\
12.346 & 730 & 17.78 & 0.13 & $R$ \\
12.980 & 759 & 17.68 & 0.12 & $R$ \\
13.239 & 759 & 17.82 & 0.16 & $R$ \\
13.971 & 997 & 18.21 & 0.12 & $R$ \\
14.611 & 1101 & 18.32 & 0.14 & $R$ \\
33.791 & 4012 & 19.35 & 0.19 & $R$ \\
\hline
\end{tabular}

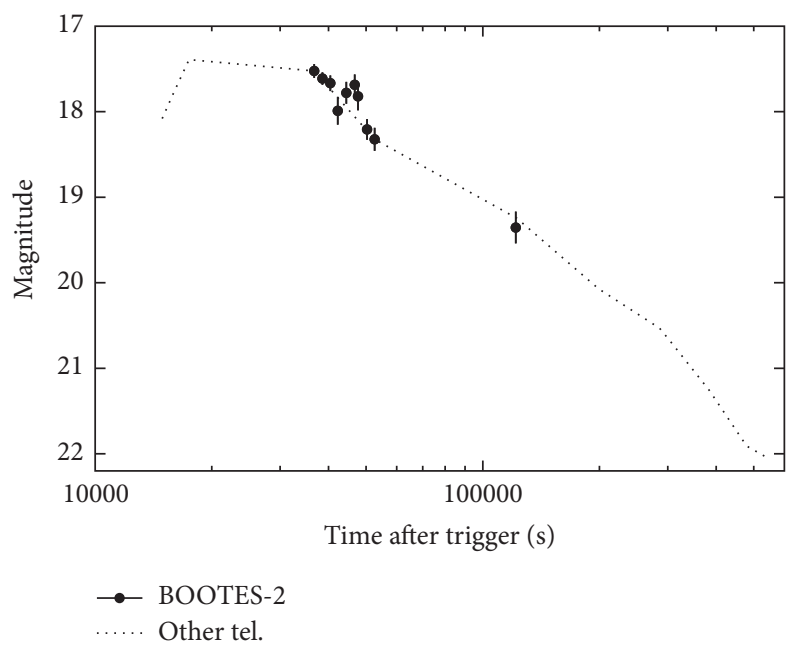

FIgURE 8: The optical light curve of GRB 100901A. The dotted line representing burst behaviour is based on observations by Gorbovskoy et al. [49], Kann et al. [51], and Rumyantsev et al. [52].

detected also the following night with $R=19.35$; see Table 10 and Figure 8.

GRB 101112A. An INTEGRAL-localized burst [53] was also detected by Fermi-GBM [54], Konus-Wind [55], and SwiftXRT [56]. Optical afterglow was discovered independently by BOOTES-2 and Liverpool Telescope [57]. It was detected also in radio [58]

BOOTES- 2 reacted to the GRB 101112A and started to observe $47 \mathrm{~s}$ after the GRB. A set of $3 \mathrm{~s}$ exposures was taken, but due to technical problems with the mount a significant amount of observing time was lost. An optical afterglow was discovered and reported [59]. The optical light curve exhibited first a decay, then a sudden rise to a peak at about $800 \mathrm{~s}$ after the trigger, and finally a surprisingly fast decay with $\alpha \simeq-4$. This behaviour seemed more like an optical flare than a "proper" GRB afterglow, but there does not seem to be contemporaneous high-energy data to make a firm statement; see Table 11 and Figure 9.

GRB 110205A (A Very Long and Bright Burst by Swift). Detected also by Konus-Wind and Suzaku-WAM, optical

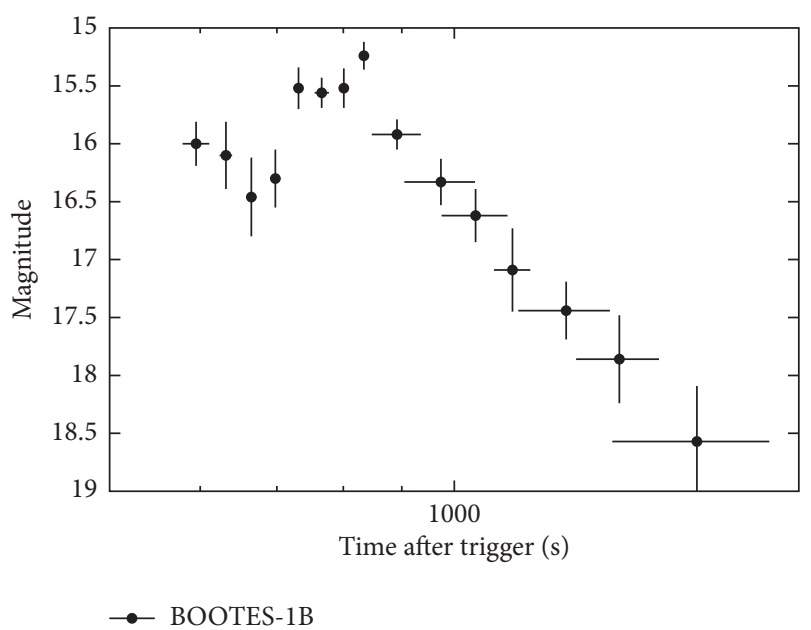

FIgURE 9: The optical light curve of GRB 101112A.

TABLE 11: GRB 101112A: observing log of BOOTES-2.

\begin{tabular}{lcccc}
\hline$\Delta T[\mathrm{~s}]$ & exp $[\mathrm{s}]$ & mag & dmag & Filter \\
\hline 595.0 & 16 & 16.00 & 0.19 & $r^{\prime}$ \\
631.8 & 8 & 16.10 & 0.29 & $r^{\prime}$ \\
664.9 & 7 & 16.46 & 0.34 & $r^{\prime}$ \\
697.8 & 7 & 16.30 & 0.25 & $r^{\prime}$ \\
731.0 & 7 & 15.52 & 0.18 & $r^{\prime}$ \\
766.1 & 11 & 15.56 & 0.13 & $r^{\prime}$ \\
800.9 & 7 & 15.52 & 0.17 & $r^{\prime}$ \\
833.8 & 7 & 15.24 & 0.12 & $r^{\prime}$ \\
891.2 & 44 & 15.92 & 0.13 & $r^{\prime}$ \\
973.7 & 69 & 16.33 & 0.20 & $r^{\prime}$ \\
1044.0 & 69 & 16.62 & 0.23 & $r^{\prime}$ \\
1124.2 & 41 & 17.09 & 0.36 & $r^{\prime}$ \\
1252.7 & 115 & 17.44 & 0.25 & $r^{\prime}$ \\
1393.8 & 116 & 17.86 & 0.38 & $r^{\prime}$ \\
1629.5 & 255 & 18.57 & 0.48 & $r^{\prime}$ \\
\hline
\end{tabular}

afterglow peaked at $R \sim 14.0$, with extensive multiwavelength follow-up; $z=2.22$ "Textbook burst" [13, 60].

BOOTES-1B reacted automatically to the Swift trigger. First $10 \mathrm{~s}$ unfiltered exposure was obtained $102 \mathrm{~s}$ after the beginning of the GRB (with $T_{90}=257 \mathrm{~s}$ ), that is, while the gamma-ray emission was still taking place. After taking 18 images, the observatory triggered on a false alarm from the rain detector, which caused the observation to be stopped for 20 minutes. After resuming the observation, $3 \times 30$ s images were obtained and another false alert struck over. This alert was remotely overridden by Kubánek, so that all 20 minutes was not lost. From then on, the observation continued until sunrise. The afterglow is well detected in the images until 2.2 hours after the GRB. 16 photometric points from combined images were eventually published.

BOOTES- 2 started observations $15 \mathrm{~min}$ after the trigger, clearly detecting the afterglow in $R$-band until 3.2 hours after the burst. 13 photometric points were obtained. The delay was 


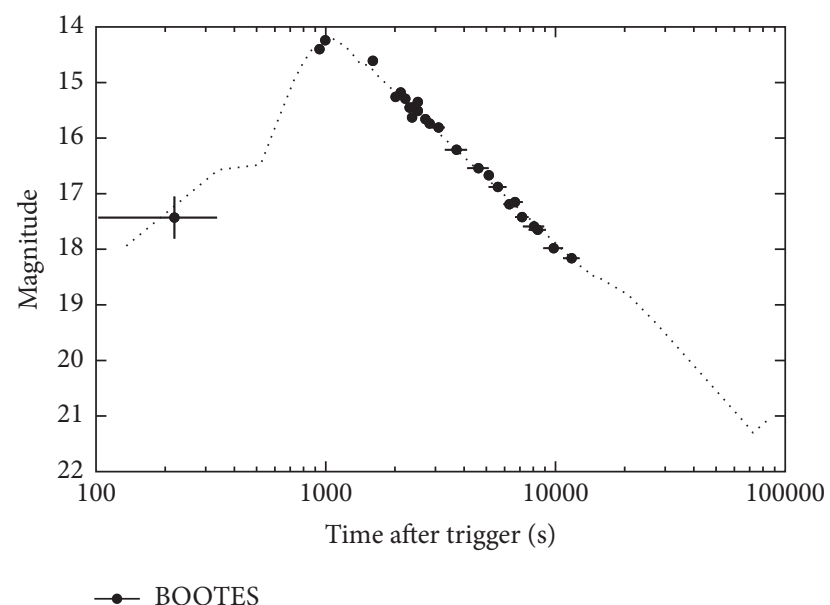

Figure 10: The optical light curve of GRB 110205A.

TABLE 12: GRB 110213A: observing log of BOOTES-1B.

\begin{tabular}{lcccc}
\hline$\Delta T[\mathrm{~h}]$ & $\exp [\mathrm{s}]$ & mag & dmag & Filter \\
\hline 15.5 & $100 \times 30$ & 18.29 & 0.30 & Clear \\
\hline
\end{tabular}

caused by technical problems. BOOTES observations of this GRB are included in Zheng et al. [13]; see Figure 10.

GRB 110213A. A bright burst was detected by Swift; it was detected also by Konus-Wind and Fermi-GBM. Optical afterglow is $R \sim 14.6$, with extensive follow-up [61].

BOOTES-1B started to observe 15 hours after the GRB (the position was below horizon at the time of the trigger) and continued for an hour; eventually, $100 \times 30 \mathrm{~s}$ unfiltered images were combined; the OT brightness calibrated against USNO-A2 is $18.3 \pm 0.3$ at the exposure midtime of $15.5 \mathrm{~h}$ after the GRB trigger; see Table 12.

GRB 120326A (A Swift-Detected Burst). Afterglow was discovered by Tarot [62]. It is long-lived optical emission; redshift is $z=1.78$ by GTC. It was detected also by Fermi-GBM and Suzaku-WAM (see [63] and the references therein).

At BOOTES-1B the mount failed, because of the serial port communication failure. After a manual recovery, 40 minutes after the GRB, images were taken in hope for a detection, but the counterpart with the brightness of $R \sim 19.5$ was detected only at about $2 \sigma$ level.

GRB 120327A. A bright burst by Swift with an afterglow is discovered by UVOT [64]. Redshift is $z=2.813$ [65]. Extensive optical follow-up was carried out.

BOOTES-1B reacted in $41 \mathrm{~min}$ (similar failure as the day before: the mount failed, because of the serial port communication failure), obtaining a series of $20 \mathrm{~s}$ exposures. These images were combined to get $600 \mathrm{~s}$ effective exposures and permitted detection of the afterglow on six such images. The brightness was decaying from $R=17.5$ to $R=18.6$; see Table 13.
TABLE 13: GRB 120327A: observing log of BOOTES-1B.

\begin{tabular}{lcccc}
\hline$\Delta T[\mathrm{~h}]$ & exp $[\mathrm{s}]$ & mag & dmag & Filter \\
\hline 0.955 & 654 & 17.50 & 0.12 & Clear \\
1.140 & 674 & 17.65 & 0.12 & Clear \\
1.337 & 748 & 17.82 & 0.13 & Clear \\
1.533 & 660 & 18.24 & 0.21 & Clear \\
1.718 & 673 & 18.17 & 0.21 & Clear \\
1.905 & 656 & 18.59 & 0.29 & Clear \\
\hline
\end{tabular}

TABLE 14: GRB 121024A: observing log of BOOTES-1B.

\begin{tabular}{lcccc}
\hline$\Delta T[\mathrm{~h}]$ & $\exp [\mathrm{s}]$ & mag & dmag & Filter \\
\hline 0.900 & 1200 & 18.2 & 0.5 & Clear \\
\hline
\end{tabular}

All-sky camera at BOOTES-1 (CASSANDRA1) covered the event in real time and detected nothing down to $R \sim 7.5$ (Zanioni et al. in prep.).

GRB 121001A. A bright and long Swift-detected GRB was originally designated as possibly galactic [66]. Afterglow was discovered by Andreev et al. [67].

BOOTES-2 observed this trigger starting $32 \mathrm{~min}$ after the trigger. An optical afterglow is detected in $I$-band with $I \sim$ 19.7 (Vega) for a sum of images between 20:49 and 21:52 UT [68].

GRB 121024A. It is a bright Swift-detected GRB with a bright optical afterglow $[69,70]$. It was detected also in radio [71]. Redshift is $z=2.298$ by Tanvir et al. [72].

BOOTES-1B observed the optical afterglow of GRB 121024A. The observations started 40 minutes after the GRB trigger. The sum of 20 minutes of unfiltered images with a mean integration time 54 minutes after the GRB shows a weak detection of the optical afterglow with magnitude $R=$ $18.2 \pm 0.5$ [73]; see Table 14 .

GRB 130418A. It is a bright and long burst with a well-detected optical afterglow somewhat peculiarly detected after a slew by Swift [74]. Observation by Konus-Wind showed that the burst started already 218 s before Swift triggered [75]. Redshift is $z=1.218$ by de Ugarte Postigo et al. [76].

BOOTES-2 obtained a large set of unfiltered, $r^{\prime}$-band and $i^{\prime}$-band images starting $1.5 \mathrm{~h}$ after the trigger. The optical afterglow is well detected in the images. The light curve is steadily decaying with the power-law index of $\alpha=-0.93 \pm$ 0.06 , with the exception of the beginning, where there is a possible flaring with peak about 0.25 mag brighter than the steady power-law; see Table 15 and Figure 11.

GRB 130505A. A bright and intense GRB with a 14 mag optical afterglow was detected by Swift [77]. Redshift is $z=2.27$ as reported by Tanvir et al. [78].

BOOTES-2 obtained the first image of this GRB $11.94 \mathrm{~h}$ after the trigger. A set of $60 \mathrm{~s}$ exposures was obtained. Combining the first hour of images taken, we clearly detect the optical afterglow, and using the calibration provided by Kann et al. [79], we measure $R_{C}=19.26 \pm 0.06$; see Table 16 . 
TABLE 15: GRB 130418A: observing log of BOOTES-1B and BOOTES-2.

\begin{tabular}{lcccc}
\hline$\Delta T[\mathrm{~h}]$ & exp $[\mathrm{s}]$ & mag & dmag & Filter \\
\hline 1.514 & $3 \times 15 \mathrm{~s}$ & 17.09 & 0.08 & Clear \\
1.529 & $3 \times 15 \mathrm{~s}$ & 16.95 & 0.07 & Clear \\
1.544 & $3 \times 15 \mathrm{~s}$ & 16.90 & 0.06 & Clear \\
1.558 & $3 \times 15 \mathrm{~s}$ & 16.62 & 0.07 & Clear \\
1.573 & $3 \times 15 \mathrm{~s}$ & 17.03 & 0.07 & Clear \\
1.590 & $4 \times 15 \mathrm{~s}$ & 16.92 & 0.06 & Clear \\
1.610 & $4 \times 15 \mathrm{~s}$ & 17.04 & 0.07 & Clear \\
1.749 & $7 \times 15 \mathrm{~s}$ & 17.22 & 0.05 & Clear \\
1.865 & $60 \mathrm{~s}$ & 16.92 & 0.18 & $r^{\prime}$ \\
1.884 & $4 \times 15 \mathrm{~s}$ & 17.34 & 0.09 & Clear \\
2.054 & $7 \times 15 \mathrm{~s}$ & 17.45 & 0.07 & Clear \\
2.089 & $7 \times 15 \mathrm{~s}$ & 17.46 & 0.06 & Clear \\
2.209 & $6 \times 15 \mathrm{~s}$ & 17.47 & 0.07 & Clear \\
2.326 & $6 \times 15 \mathrm{~s}$ & 17.56 & 0.08 & Clear \\
2.444 & $6 \times 15 \mathrm{~s}$ & 17.71 & 0.09 & Clear \\
2.562 & $6 \times 15 \mathrm{~s}$ & 17.68 & 0.08 & Clear \\
2.798 & $22 \times 60 \mathrm{~s}$ & 17.40 & 0.04 & $i^{\prime}$ \\
3.061 & $15 \times 60 \mathrm{~s}$ & 17.90 & 0.09 & $r^{\prime}$ \\
3.333 & $15 \times 60 \mathrm{~s}$ & 17.98 & 0.09 & $r^{\prime}$ \\
3.604 & $15 \times 60 \mathrm{~s}$ & 17.90 & 0.09 & $r^{\prime}$ \\
3.866 & $15 \times 60 \mathrm{~s}$ & 18.05 & 0.11 & $r^{\prime}$ \\
4.130 & $15 \times 60 \mathrm{~s}$ & 18.53 & 0.19 & $r^{\prime}$ \\
4.449 & $20 \times 60 \mathrm{~s}$ & 18.42 & 0.14 & $r^{\prime}$ \\
4.808 & $20 \times 60 \mathrm{~s}$ & 18.61 & 0.23 & $r^{\prime}$ \\
\hline & & & &
\end{tabular}

TABLE 16: GRB 130505A: observing log of BOOTES-2.

\begin{tabular}{lcccc}
\hline$\Delta T[\mathrm{~h}]$ & $\exp [\mathrm{s}]$ & mag & dmag & Filter \\
\hline 12.488 & $51 \times 60 \mathrm{~s}$ & 19.26 & 0.06 & Clear
\end{tabular}

GRB 130606A. A high-redshift GRB was detected by Swift [80], optical afterglow was discovered by BOOTES-2, and redshift is $z=5.9$ by GTC [81].

BOOTES-2 reaction to this GRB alert was actually a failure; the system did not respond as well as it should and it had to be manually overridden to perform the observations. The first image has therefore been taken as late as 13 minutes after the trigger. These observations led to a discovery of a bright afterglow not seen by Swift-UVOT and prompted spectroscopic observations by $10.4 \mathrm{~m}$ GTC, which show redshift of this event to be $z=5.9135$. Overall, 14 photometric points in $i^{\prime}$-band and 7 in $z^{\prime}$-band were obtained [81]; see Figure 12.

\section{Summary}

Eleven years of BOOTES-1B and BOOTES-2 GRB follow-up history are summarised in the textual and tabular form. Each GRB is given a short introductory paragraph as a reminder of the basic optical properties of the event. Although we do not discuss the properties in other wavelengths, we try to include a comprehensive reference of literature relevant to

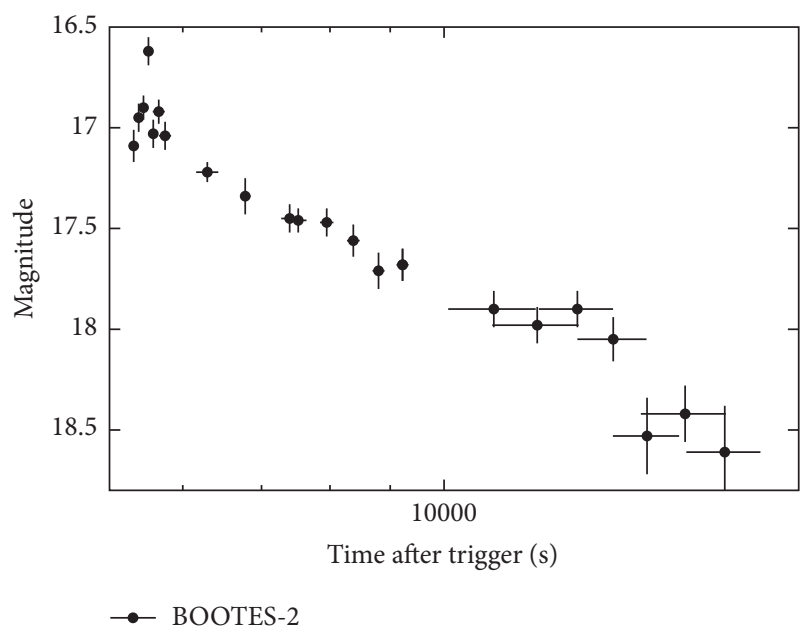

FIGURE 11: The optical light curve of GRB 130418A.

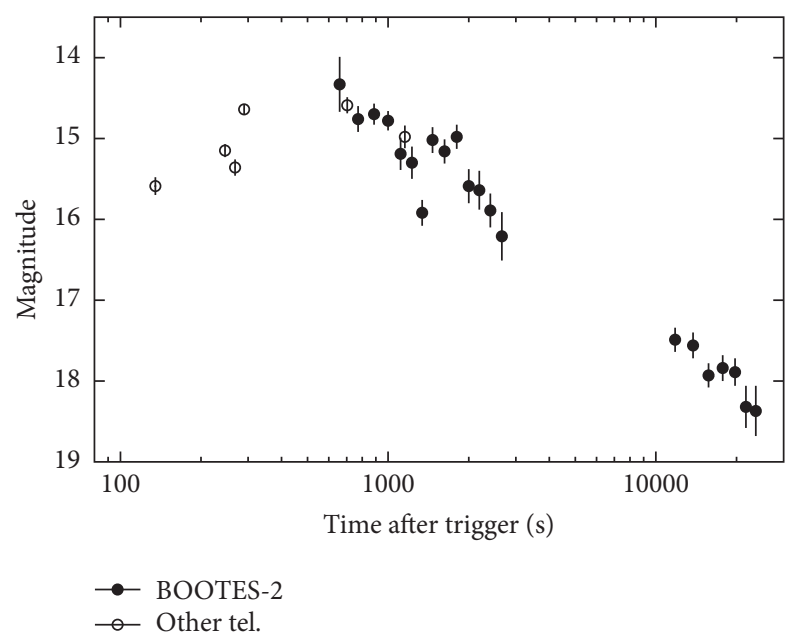

FIgURE 12: The optical light curve of GRB 130606A. $i^{\prime}$-band points were shifted 2.4 mag up to match with the $z^{\prime}$-band points.

each burst. One by one, we show all the successful follow-ups that these telescopes have performed during the first ten years of the Swift era and the transition of the BOOTES network to the RTS-2 [14] observatory control system, first installed at BOOTES-2 in 2003 and made definitive during the summer of 2004.

The BOOTES telescopes, in spite of their moderate apertures $(\leqslant 60 \mathrm{~cm})$, have proven to detect a significant number of afterglows-together over 20, contributing to the understanding of the early GRB phase.

\section{Competing Interests}

The authors declare that they have no competing interests.

\section{Acknowledgments}

The authors appreciate the auspices of INTA, IHSMUMA/CSIC, and UMA as well as the financial support by 
the Junta de Andaluca and the Spanish Ministry of Economy and Competitiveness through the Research Projects P07-TIC-03094, P12-TIC2839, AYA 2009-14000-C03-01, AYA 2010-39727-C03-01, and AYA-2015-71718-R. Martin Jelínek was supported by the postdoctoral fellowship of the Czech Academy of Sciences. This study was carried out in the framework of the Unidad Asociada IAA-CSIC at the Group of Planetary Science of ETSI-UPV/EHU. This work was supported by the Ikerbasque Foundation for Science. The Czech CVUT FEL team acknowledges the support by GA CR Grant 13-33324S.

\section{References}

[1] R. W. Klebesadel, I. B. Strong, and R. A. Olson, "Observations of gamma-ray bursts of cosmic origin," The Astrophysical Journal, vol. 182, pp. L85-L88, 1973.

[2] E. Costa, F. Frontera, J. Heise et al., "Discovery of an X-ray afterglow associated with the $\gamma$-ray burst of 28 February 1997," Nature, vol. 387, no. 6635, pp. 783-785, 1997.

[3] J. van Paradijs, P. J. Groot, T. Galama et al., "Transient optical emission from the error box of the $\gamma$-ray burst of 28 february 1997," Nature, vol. 386, no. 6626, pp. 686-689, 1997.

[4] J. L. Racusin, S. V. Karpov, M. Sokolowski et al., "Broadband observations of the naked-eye big $\gamma$-ray burst GRB 080319B," Nature, vol. 455, pp. 183-188, 2008.

[5] A. J. Castro-Tirado, M. Jelínek, T. J. Mateo Sanguino, and A. De Ugarte Postigo, "BOOTES: a stereoscopic robotic ground support facility," Astronomische Nachrichten, vol. 325, article 679, 2004.

[6] M. Jelínek, A. J. Castro-Tirado, A. D. U. Postigo et al., "Four years of real-time GRB followup by BOOTES-1B (2005-2008)," Advances in Astronomy, vol. 2010, Article ID 432172, 10 pages, 2010.

[7] O. Rabaza, M. Jelínek, A. J. Castro-Tirado et al., "Compact low resolution spectrograph, an imaging and long slit spectrograph for robotic telescopes," Review of Scientific Instruments, vol. 84, no. 11, Article ID 114501, 2013.

[8] L. Resmi, K. Misra, G. Jóhannesson et al., "Comprehensive multiwavelength modelling of the afterglow of GRB 050525A," Monthly Notices of the Royal Astronomical Society, vol. 427, no. 1, pp. 288-297, 2012.

[9] J. Sollerman, J. P. U. Fynbo, J. Gorosabel et al., "The nature of the X-ray flash of August 24 2005," A\&A, vol. 466, no. 3, pp. 839-846, 2007.

[10] J. B. Haislip, M. C. Nysewander, D. E. Reichart et al., "A photometric redshift of $z=6.39 \pm 0.12$ for GRB 050904," Nature, vol. 440, pp. 181-183, 2006.

[11] M. Jelínek, J. Gorosabel, A. J. Castro-Tirado et al., "BOOTES observation of GRB080603B," Acta Polytechnica, vol. 52, Article ID 010000, 2012.

[12] M. Jelínek, E. Gómez Gauna, and A. J. Castro-Tirado, "Photometric observations of GRB 080605 by Bootes-1b and Bootes-2," in Gamma-Ray Bursts: 15 Years of GRB Afterglows, A. J. CastroTirado, J. Gorosabel, and I. H. Park, Eds., vol. 61, pp. 475-477, EAS Publications Series, 2013.

[13] W. Zheng, R. F. Shen, T. Sakamoto et al., "Panchromatic observations of the textbook GRB 110205A: constraining physical mechanisms of prompt emission and afterglow," The Astrophysical Journal, vol. 751, no. 2, Article ID 90, 21 pages, 2012.
[14] P. Kubánek, M. Jelínek, M. Nekola et al., "RTS2-remote telescope system, 2nd version," in Proceedings of the Gamma-Ray Bursts: 30 Years of Discovery, vol. 727 of AIP Conference Proceedings, p. 753, Sante Fe, NM, USA, 2004.

[15] A. J. Blustin, D. Band, S. Barthelmy et al., "Swift panchromatic observations of the bright gamma-ray burst GRB 050525a," The Astrophysical Journal, vol. 637, no. 2, p. 901, 2006.

[16] M. Della Valle, D. Malesani, J. S. Bloom et al., "Hypernova signatures in the late rebrightening of GRB 050525A," The Astrophysical Journal Letters, vol. 642, no. 2, pp. L103-L106, 2006.

[17] A. de Ugarte Postigo, M. Jelínek, J. Gorosabel et al., "GRB 050525A: bootes simultaneous optical observations," GCN Circular 3480, 2005.

[18] J. Norris, L. Barbier, D. Burrows et al., "GRB 050822C: swift detection of a bright burst," GCN Circular 4013, 2005.

[19] H. Krimm, L. Barbier, S. Barthelmy et al., "GCN circular," Tech. Rep. 4020, 2005.

[20] G. Crew, G. Ricker, J.-L. Atteia et al., "HETE fregate observations of GRB 050922C," GCN Circular 4021, 2005.

[21] P. Jakobsson, J. P. U. Fynbo, D. Paraficz et al., "GRB 050922C: refined redshift," GCN Circular 4029, 2005.

[22] N. Mirabal, J. P. Halpern, S. Tonnesen et al., "GRB 051109A: a shallow optical afterglow decay," in Proceedings of the American Astronomical Society Meeting, vol. 207, American Astronomical Society, 2006, Abstract 210.02.

[23] E. Fenimore, L. Angelini, L. Barbier et al., "GRB 051109: SwiftBAT refined analysis," GCN Circular 4217, 2005.

[24] R. Quimby, D. Fox, P. Hoeich, B. Roman, and J. C. Wheeler, "GRB 051109: HET optical spectrum and absorption redshift," GCN Circular 4221, 2005.

[25] M. Jelínek, A. de Ugarte Postigo, A. J. Castro-Tirado et al., "GRB 051109a: bootes R \& I-band detection of the early afterglow," GCN Circular 4227, 2005.

[26] N. Mirabal, J. Halpern, S. Tonnesen, J. Eastman, and J. Prieto, 2005, http://user.astro.columbia.edu/ jules/grb/051109a/.

[27] F. Yuan, E. S. Rykoff, B. E. Schaefer et al., Prompt Optical Observations of GRB 080330 and GRB 080413A, vol. 1065 of American Institute of Physics Conference Series, American Institute of Physics, 2008, Edited by Y.-F. Huang, Z.-G. Dai, \& B. Zhang.

[28] J. Mao, C. Guidorzi, C. Markwardt et al., "Swift Observation of GRB 080330," GCN Report 132, 2008.

[29] F. E. Marshall, S. D. Barthelmy, D. N. Burrows et al., "Final swift observations of GRB 080413A," GCN Report 129, 2008.

[30] P. Kubánek, M. Jelínek, J. Gorosabel et al., "GCN circular," Tech. Rep. 7603, 2008.

[31] C. Guidorzi, M. Stamatikos, W. Landsman et al., "Swift Observations of GRB 080430," GCN Report 139, 2008.

[32] J. Aleksić, H. Anderhub, L. A. Antonelli et al., "MAGIC observation of the GRB 080430 afterglow," Astronomy \& Astrophysics, vol. 517, article A5, 2010.

[33] M. Jelínek, P. Kubánek, J. Gorosabel et al., "A decade of GRB follow-up by BOOTES in Spain," 9 Circular 7648, 2008.

[34] A. Rau, A. V. Kienlin, K. Hurley, and G. G. Lichti, “The 1st INTEGRAL SPI-ACS gamma-ray burst catalogue," Astronomy and Astrophysics, vol. 438, no. 3, pp. 1175-1183, 2005.

[35] V. Mangano, A. Parsons, T. Sakamoto et al., "Swift observation of GRB 080603B,” GCN Report 144, 2008. 
[36] B. Sbarufatti, A. Parsons, T. Sakamoto et al., "Swift observation of GRB 080605," GCN Report, vol. 142, 2008.

[37] T. Kruhler, J. P. U. Fynbo, S. Geier et al., "The metal-enriched host of an energetic $\gamma$-ray burst at $z \approx 1.6, "$ Astronomy \& Astrophysics, vol. 546, article A8, 2012.

[38] T. Zafar, D. Watson, Á. Elíasdóttir et al., "The properties of the 2175 å extinction feature discovered in GRB afterglows," The Astrophysical Journal, vol. 753, no. 1, p. 82, 2012.

[39] V. Rumyantsev and A. Pozanenko, "GRB 080605: optical observations," GRB Coordinates Network 7857, 2008.

[40] J. Mao, R. Margutti, T. Sakamoto et al., "Swift observations of GRB 090313," GCN Report 204, 2009.

[41] A. de Ugarte Postigo, P. Goldoni, C. C. Thone et al., "GRB 090313: X-shooter's first shot at a gamma-ray burst," $A \& A$, vol. 513, article A42, 2010.

[42] A. Melandri, S. Kobayashi, C. G. Mundell et al., "GRB 090313 and the origin of optical peaks in $\gamma$-ray burst light curves: implications for lorentz factors and radio flares," The Astrophysical Journal, vol. 723, no. 2, p. 1331, 2010.

[43] J. R. Cummings, A. P. Beardmore, and P. Schady, "Swift observations of GRB 090813," GCN Report 240, 2009.

[44] J. Gorosabel, V. Terron, M. Fernandez et al., "GRB 090813: optical candidate from 1.23 m CAHA telescope," GCN Circular 9782, 2009.

[45] F. E. Marshall, L. A. Antonelli, D. N. Burrows et al., "The late peaking afterglow of GRB 100418A," The Astrophysical Journal, vol. 727, no. 2, p. 132, 2011.

[46] A. de Ugarte Postigo, C. C. Thone, P. Goldoni, and J. P. U. Fynbo, "Time resolved spectroscopy of GRB 100418A and its host galaxy with X-shooter," Astronomische Nachrichten, vol. 332, no. 3, pp. 297-298, 2011.

[47] A. Moin, P. Chandra, J. C. A. Miller-Jones et al., "Radio observations of GRB 100418a: test of an energy injection model explaining long-lasting grb afterglows," The Astrophysical Journal, vol. 779, no. 2, p. 105, 2013.

[48] S. Immler, T. Sakamoto, K. L. Page et al., "Swift observations of GRB 100901A," GCN Report 304, 2010.

[49] E. S. Gorbovskoy, G. V. Lipunova, V. M. Lipunov et al., "Prompt, early and afterglow optical observations of five $\gamma$-ray bursts: GRB 100901A, GRB 100902A, GRB 100905A, GRB 100906A and GRB 101020A," MNRAS, vol. 421, no. 3, pp. 1874-1890, 2012.

[50] O. E. Hartoog, K. Wiersema, P. M. Vreeswijk et al., "The hostgalaxy response to the afterglow of GRB 100901A," Monthly Notices of the Royal Astronomical Society, vol. 430, no. 4, pp. 2739-2754, 2013.

[51] D. A. Kann, U. Laux, and B. Stecklum, "GRB 100901A: TLS observations, SDSS calibration, decay slope," GCN Circular 11236, 2010.

[52] V. Rumyantsev, D. Shakhovkoy, and A. Pozanenko, "GRB 100901A: CrAO optical observation," GCN Circular 11255, 2010.

[53] D. Gotz, S. Mereghetti, A. Paizis et al., "GRB 101112A: a long GRB detected by INTEGRAL," GCN Circular 11396, 2010.

[54] A. Goldstein, "GRB 101112A: Fermi GBM detection," GCN Circular 11403, 2010.

[55] S. Golenetskii, R. Aptekar, D. Frederiks et al., "Konus-wind observation of GRB 101112A," GCN Circular 11400, 2010.

[56] P. A. Evans and H. A. Krimm, "GRB 101112A-XRT source detection/analysis," GCN Circular 11399, 2010.

[57] C. Guidorzi, R. J. Smith, C. G. Mundell et al., "GRB101112A: Liverpool telescope afterglow candidate," GCN Circular 11397, 2010.
[58] P. Chandra, D. A. Frail, and S. B. Cenko, "Possible detection of INTEGRAL burst GRB 101112A by the EVLA," GCN Circular 11404, 2010.

[59] A. de Ugarte Postigo, P. Kubánek, J. C. Tello et al., "GRB 101112A: BOOTES-2/TELMA optical afterglow candidate," GCN Circular 11398, 2010.

[60] B. Gendre, J. L. Atteia, M. Boer et al., "GRB 110205A: anatomy of a long gamma-ray burst," The Astrophysical Journal, vol. 748, no. 1, Article ID 59, 2012.

[61] V. D’Elia, G. Stratta, N. P. M. Kuin et al., “Swift observation of GRB 110213A," GCN Report 323, 2011.

[62] A. Klotz, B. Gendre, M. Boer, and J. L. Atteia, "GRB 120326A: TAROT calern observatory afterglow optical detection," GCN Circular 13107, 2012.

[63] M. H. Siegel, N. P. M. Kuin, S. Holland et al., "Swift observations of GRB 120326A,” GCN Report 409, 2013.

[64] B. Sbarufatti, S. D. Barthelmy, N. Gehrels et al., "GRB 120327A: swift detection of a burst with an optical counterpart," GCN Circular 13123, 2012.

[65] V. D'Elia, "VLT/X-shooter absorption spectroscopy of the GRB 120327a afterglow," in EAS Publications Series, A. J. CastroTirado, J. Gorosabel, and I. H. Park, Eds., vol. 61 of EAS Publications Series, pp. 247-249, 2013.

[66] V. D'Elia, J. R. Cummings, M. Stamatikos et al., "Swift observations of GRB 121001A," GCN Report 392, 2012.

[67] M. Andreev, A. Sergeev, and A. Pozanenko, "GRB 121001A: possible optical counterpart," GCN Circular 13833, 2012.

[68] J. C. Tello, R. Gimeno, J. Gorosabel et al., "Swift trigger 535026: optical decay confirmation with IAC80 and BOOTES2/TELMA," GCN Circular 13835, 2012.

[69] C. Pagani, S. D. Barthelmy, W. H. Baumgartner et al., "GRB 121024A: swift detection of a burst with an optical counterpart," GCN Circular 13886, 2012.

[70] A. Klotz, B. Gendre, M. Boer, and J. L. Atteia, “GRB 121024A: TAROT calern observatory optical detection of a bright counterpart," GCN Circular 13887, 2012.

[71] T. Laskar, A. Zauderer, and E. Berger, "GRB 121024A: EVLA detection," GCN Circular 13903, 2012.

[72] N. R. Tanvir, J. P. U. Fynbo, A. Melandri et al., "GRB 121024A: VLT/X-shooter redshift,” GCN Circular 13890, 2012.

[73] M. Jelínek, A. J. Castro-Tirado, and J. Gorosabel, “GRB 121024A: BOOTES-1B optical detection,” GCN Circular 13888, 2012.

[74] M. de Pasquale, W. H. Baumgartner, A. P. Beardmore et al., "GRB 130418A: swift detection of a burst with an optical counterpart," GCN Circular 14377, 2013.

[75] S. Golenetskii, R. Aptekar, D. Frederiks et al., "Konus-wind observation of GRB 130418A," GCN Circular 14417, 2013.

[76] A. de Ugarte Postigo, C. C. Thoene, J. Gorosabel et al., "GRB 130418A: redshift from 10.4 m GTC," GCN Circular 14380, 2013.

[77] J. K. Cannizzo, S. D. Barthelmy, J. R. Cummings, A. Melandri, and M. de Pasquale, "Swift observations of GRB 130505A," GCN Report 429, 2013.

[78] N. R. Tanvir, A. J. Levan, T. Matulonis, and A. B. Smith, "GRB 130505A-Gemini-N/GMOS redshift determination,” GCN Circular 14567, 2013.

[79] D. A. Kann, B. Stecklum, and F. Ludwig, "GRB 130505A: tautenburg afterglow observations," GCN Circular 14593, 2013.

[80] T. N. Ukwatta, M. Stamatikos, A. Maselli et al., "Swift observations of GRB 130606A," GCN Report 444, 2013. 
[81] A. J. Castro-Tirado, R. Sánchez-Ramírez, S. L. Ellison et al., "GRB 130606A within a sub-DLA at redshift 5.91," http://arxiv .org/abs/1312.5631. 

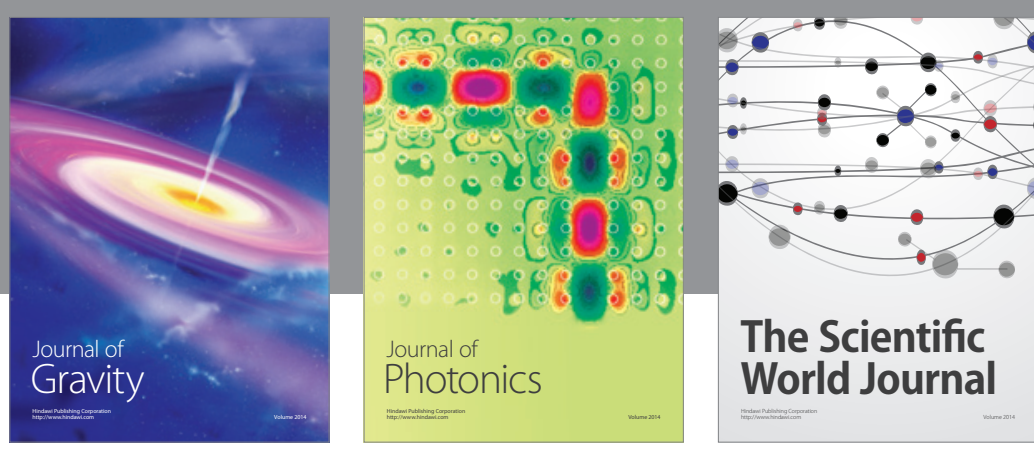

The Scientific World Journal
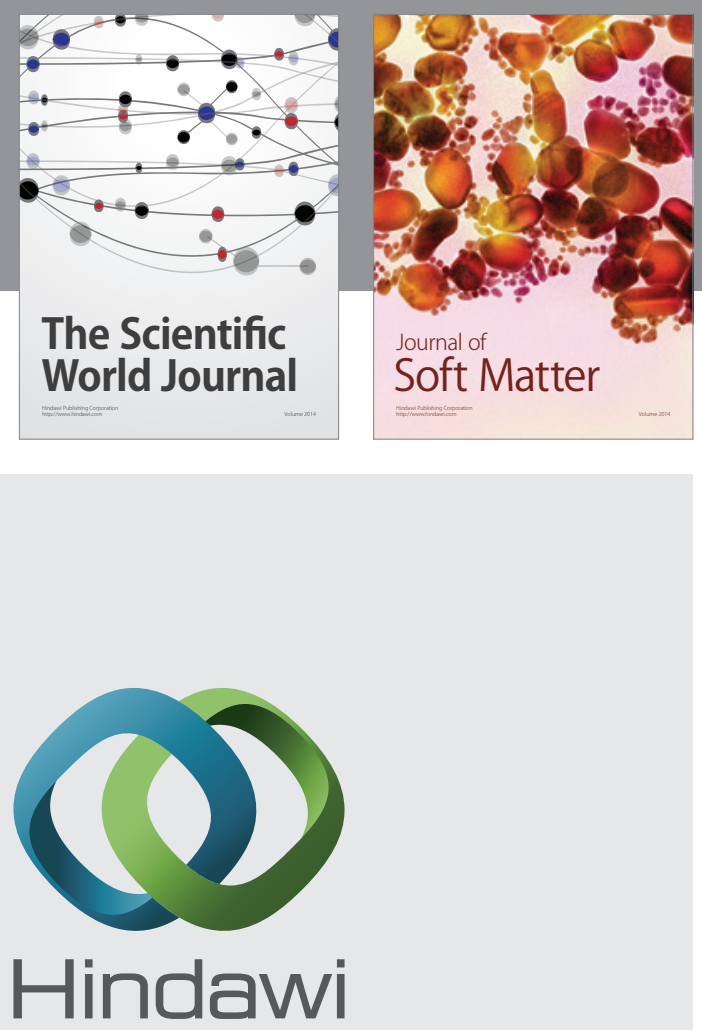

Submit your manuscripts at

http://www.hindawi.com

nternational Journal of

Statistical Mechanics
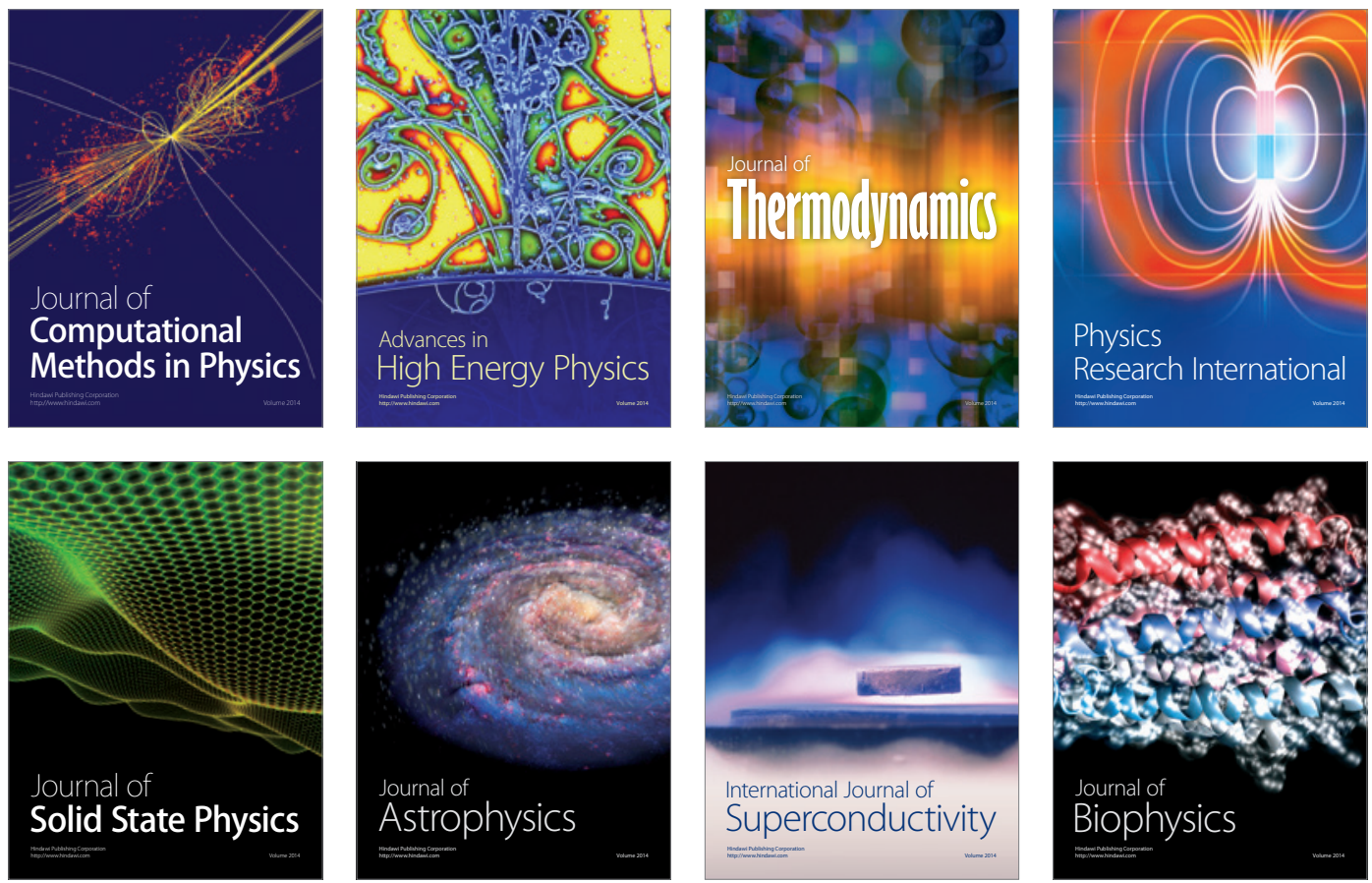
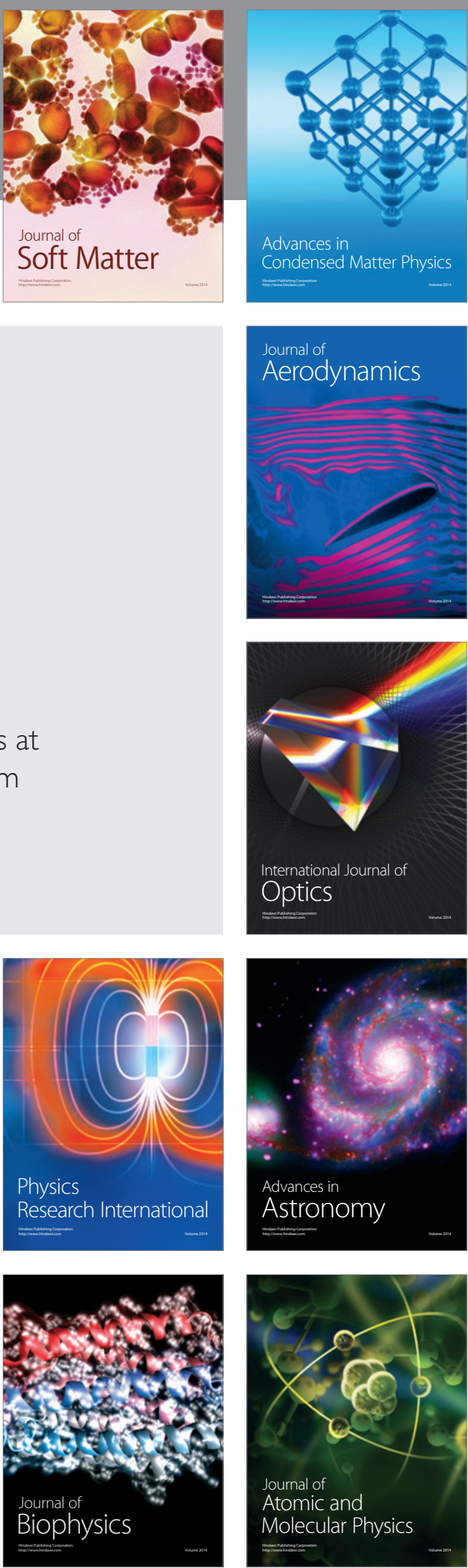